\title{
Impacto nutricional do programa de alimentação do trabalhador no Brasil ${ }^{1}$
}

\author{
Iracema Santos Veloso ${ }^{2}$ e Vilma Sousa Santana ${ }^{3}$
}

RESUMO Objetivos. O programa de alimentação do trabalhador do Ministério do Trabalho e Emprego do Brasil destina-se a garantir alimentação adequada a trabalhadores de baixa renda. Neste estudo, avaliou-se o impacto nutricional do programa, que atende cerca de 10 milhões de pessoas no país.

Métodos. Este é um estudo de coorte dinâmica retrospectiva com 8454 trabalhadores residentes no Estado da Bahia, Brasil. Utilizaram-se dados de prontuários médicos on-line pertencentes a uma organização que presta serviços de monitoramento da saúde de trabalhadores a um grande número de empresas em todo o Estado da Bahia. Foram utilizados dados demográficos e nutricionais (peso e pré-obesidade). Informações adicionais foram obtidas junto às empresas em entrevistas telefônicas.

Resultados. Ser beneficiário do programa foi associado positivamente a aumento de peso (razão de densidade de incidência ajustada por idade e sexo $=2,21$; intervalo de confiança de $95 \%=1,78-2,75)$. Não houve associação estatisticamente significativa entre participar no programa de alimentação e pré-obesidade. O risco de aumento de peso foi maior entre os trabalhadores eutróficos, pré-obesos ou de baixo nível socioeconômico no início do seguimento $(\mathrm{P}<0,05)$.

Conclusões. Os resultados sugerem que o programa de alimentação do trabalhador tem impacto negativo sobre o estado nutricional dos trabalhadores de baixa renda. Suas estratégias, restritas a recomendações energético-protéicas, precisam ser reavaliadas para que efetivamente promovam a saúde do trabalhador.

Palavras-chave Inquéritos nutricionais, programas e políticas de nutrição e alimentação, avaliação de programas de nutrição, estado nutricional.

1 Estudo financiado pelo Conselho Nacional de Desenvolvimento Científico e Tecnológico (CNPQ Processo 522.621-96-1) e National Institutes of Health/Fogarty Foundation (Grant No. 1043 TW00827-02), mediante convênio entre a University of North Carolina at Chapel Hill, EUA, e o Instituto de Saúde Coletiva da Universidade Federal da Bahia, Brasil.

2 Universidade Federal da Bahia, Escola de $\mathrm{Nu}$ trição. Correspondência e pedidos de separatas devem ser enviados a esta autora no seguinte endereço: Rua Araújo Pinho 32, Canela, CEP 40110150, Salvador, BA, Brasil. Fone: +55-71-245-0587; fax: +55-71-237-5856; e-mail: iravel@ufba.br

3 Universidade Federal da Bahia, Instituto de Saúde Coletiva.
No Brasil, as políticas de alimentação para o trabalhador se fundamentam na idéia de que a força de trabalho é elemento chave para a produção econômica. Estas políticas se materializaram na década de 1940, com a criação do serviço de alimentação da previdência social (SAPS), que perdurou até a década de 1960. No início da década de 1970, diante do agravamento dos problemas sociais, o governo redefiniu sua estratégia de en- frentamento da crise social e sanitária que então ocorria. Programas compensatórios das desigualdades sociais voltados para as necessidades básicas dos indivíduos foram criados. Dentre estes, o Programa Nacional de Alimentação e Nutrição (PRONAN) (1) se constituiu na proposta mais abrangente para combater os problemas alimentares no país. Sua elaboração se fundamentou em dados que apontavam deficiência calórica em $67 \%$ da população, prova 
de que o principal problema alimentar no Brasil seria de natureza quantitativa. Uma de suas linhas de ação foi expandir a cobertura para trabalhadores de baixa renda. Assim, criou-se o programa de alimentação do trabalhador (PAT) (2), com o objetivo de melhorar o estado nutricional do trabalhador, aumentar sua produtividade e reduzir os acidentes de trabalho e o absenteísmo.

O PAT encontra-se sob a responsabilidade do Ministério do Trabalho e Emprego. Seus recursos provêm dos trabalhadores (20\%) e de empresas e governo (80\%), e o acesso à alimentação é viabilizado por refeições servidas no local de trabalho ou pelo fornecimento de cupons e cestas básicas. $\mathrm{O}$ PAT recomenda que as refeições maiores (almoço, jantar e ceia) devam ter no mínimo $1400 \mathrm{kcal}$, e as menores (desjejum e merenda), $300 \mathrm{kcal}$, todas com mais de $6 \%$ de composição protéicocalórica (3).

Estudos $(4,5)$ mostram que, atualmente, a situação nutricional é diferente daquela observada na época em que surgiu o PAT, sendo a pré-obesidade elevada entre trabalhadores, com estimativas de prevalência variando entre 34 e 56\%. Portanto, é possível que as recomendações do PAT não sejam mais adequadas às necessidades dos trabalhadores.

Poucas são as pesquisas para avaliação do PAT, e nenhuma delas focalizou o impacto nutricional. Neste estudo, pretendeu-se verificar o impacto do PAT no estado nutricional, medido pelo ganho de peso e desenvolvimento da pré-obesidade, e se esse impacto se modifica de acordo com as características nutricionais e socioeconômicas dos trabalhadores.

\section{MATERIAIS E MÉTODOS}

Este é um estudo de coorte retrospectiva, de população dinâmica, realizado com dados de uma organização que presta serviços de monitoramento da saúde de trabalhadores a um grande número de empresas em todo o Estado da Bahia. A população fonte consistiu em trabalhadores do setor in- dustrial, especialmente de pequenas e médias empresas, que foram examinados entre $1^{\circ}$ de janeiro de 1996 e 30 de abril de 2000. A população do estudo foi definida pelo total de pessoas atendidas pelo menos duas vezes no período do estudo para exames de admissão ao emprego, exames periódicos ou exames de demissão.

A base de dados do estudo incluiu informações selecionadas dos prontuários on-line dos trabalhadores, que contêm dados sócio-demográficos e ocupacionais, história clínica e resultados de exames laboratoriais. Foi preservada a confidencialidade tanto dos trabalhadores quanto das empresas. Dados adicionais sobre o programa de alimentação foram obtidos diretamente junto às indústrias mediante entrevistas telefônicas com informantes chaves, como gerentes administrativos ou responsáveis pelo setor de recursos humanos, que responderam a um questionário padronizado com perguntas fechadas. O protocolo da pesquisa foi aprovado pela comissão de ética em pesquisa do hospital universitário da Universidade Federal da Bahia, Brasil.

A variável programa de alimentação foi composta por três categorias: 1) PAT: empresas registradas no programa de alimentação do trabalhador do Ministério do Trabalho e Emprego; 2) outro programa: empresas que possuíam outro programa de alimentação, não registrado, oferecendo refeição, ticket ou cesta básica; 3) nenhum programa: empresa sem nenhum programa de alimentação.

As variáveis relativas ao estado nutricional foram o aumento de peso e a pré-obesidade. O aumento de peso correspondeu à diferença absoluta entre o peso registrado na última avaliação clínica em relação à primeira, enquanto que a pré-obesidade foi definida com base no índice de massa corporal (IMC, peso dividido pelo quadrado da altura) entre 25,0 e 29,9 $\mathrm{kg} / \mathrm{m}^{2}$, de acordo com a recomendação da Organização Mundial da Saúde (OMS) (6). Ainda com base nesta variável, definiram-se a magreza (IMC abaixo de $18,5 \mathrm{~kg} / \mathrm{m}^{2}$ ) e eutrofia (IMC entre 18,5 e $24,9 \mathrm{~kg} / \mathrm{m}^{2}$ ), que compõem as categorias para a análise descritiva.

As variáveis sócio-demográficas e ocupacionais foram sexo, idade em anos, escolaridade, situação conjugal e ocupação, classificada em três categorias com base no nível de qualificação exigido. O nível socioeconômico foi também definido em três faixas (conforme qualificação da ocupação e escolaridade), de acordo com os seguintes critérios: nível alto: cargos de direção e assessoria e profissionais liberais, todos com nível de escolaridade superior ou médio; nível médio: ocupações administrativas de média qualificação, escolaridade equivalente a ensino médio ou técnico; nível baixo: ocupações braçais, operadores da indústria e os demais, com escolaridade primária ou analfabetos. Os fatores de risco para doenças cardiovasculares disponíveis foram: hipertensão arterial sistólica $\geq 140 \mathrm{mmHg}$ (7), hipertrigliceridemia para valores $\geq 200 \mathrm{mg} / \mathrm{dl}$, hipercolesterolemia para colesterol total $\geq 200 \mathrm{mg} / \mathrm{dl}$, (8), hiperglicemia, glicose $>110$ mg/dl (9), hábito de fumar e consumo de bebidas alcoólicas. As características sócio-demográficas e os fatores de risco para doenças cardiovasculares foram descritos com freqüências relativas estimadas de acordo com a variável programa de alimentação.

Para a inferência estatística utilizouse o teste do qui-quadrado $\left(\chi^{2}\right)$. A taxa de incidência de aumento de peso e pré-obesidade foi estimada dividindose o número de casos novos no período pelo somatório dos tempos individuais em anos (pessoa-ano, pa). Para estimar medidas de associação brutas entre ser participante de programas de alimentação e aumento de peso e préobesidade, empregou-se a razão da taxa de incidência (RTI) e seus respectivos intervalos de confiança a 95\% (IC $95 \%$ ), estimados pelo método exato binomial. A razão de taxas de incidência foi ajustada por idade e sexo $\left(\mathrm{RTI}_{\mathrm{aj}}\right)$, por serem as variáveis ser participante de programas de alimentação e aumento de peso e pré-obesidade confundidoras potenciais para a associação em análise, já que são fatores associados com o peso corporal. Essas estimativas foram feitas com regressão 
de Poisson usando-se o Statistic Analysis System (SAS) versão 6.07 (10); os respectivos IC $95 \%$ foram calculados empregando-se a estatística de Wald. Definiu-se para o estudo um alfa de 0,05 (11).

\section{RESULTADOS}

A população de trabalhadores que havia realizado pelo menos duas avaliações no período do estudo era de 8926 trabalhadores. Este número se reduziu para 8454 após a retirada daqueles ( $n=298 ; 3,3 \%$ ) para os quais não se dispunha de informações sobre a cobertura por programa de alimentação ou peso e altura $(\mathrm{n}=174 ; 1,9 \%)$. Dentre as 374 empresas identificadas, apenas quatro (1\%) não puderam ser localizadas para a pesquisa por telefone.

O estudo mostrou que os trabalhadores de empresas com PAT ou outros programas de alimentação eram mais freqüentemente do sexo masculino, com idade acima de 36 anos, casados, com nível socioeconômico melhor e maior escolaridade e qualificação ocupacional em comparação ao grupo de trabalhadores de empresas sem nenhum programa (tabela 1). Todas as diferenças foram estatisticamente significativas para um alfa $=0,05$. A tabela 2 mostra que os trabalhadores cobertos pelo PAT apresentavam taxas mais elevadas de triglicéridos, colesterol total e glicemia ou hipertensão arterial do que os trabalhadores do grupo sem programa de alimentação.

Por outro lado, a prevalência de consumo de bebidas alcoólicas foi menor entre os trabalhadores beneficiários do PAT. A distribuição das categorias do IMC também variou de acordo com a participação em programa de alimentação $(P<0,05)$, notando-se que a pré-obesidade foi mais comum entre os trabalhadores não beneficiados $(35 \%)$ do que no grupo PAT (32\%) e no grupo beneficiado por outros programas de alimentação (29\%). Não houve variações estatisticamente significativas entre os grupos em relação ao hábito de fumar.

A taxa de incidência de aumento de peso para trabalhadores de empresas
TABELA 1. Características sócio-demográficas de 8454 trabalhadores conforme programa de alimentação ao início do estudo, Bahia, Brasil, 1996 a 2000

\begin{tabular}{|c|c|c|c|}
\hline \multirow[b]{3}{*}{ Variáveis } & \multicolumn{3}{|c|}{ Programa de alimentação } \\
\hline & $\begin{array}{c}\text { Programa de } \\
\text { alimentação do } \\
\text { trabalhador }^{2}(n=3551)\end{array}$ & Outro $(n=4078)$ & Nenhum $(n=825)$ \\
\hline & No. (\%) & No. (\%) & No. (\%) \\
\hline \multicolumn{4}{|l|}{ Sexo } \\
\hline Masculino & $2918(82,17)$ & 3138 (77) & $597(72,36)^{b}$ \\
\hline Feminino & $633(17,83)$ & $940(23)$ & $228(27,64)$ \\
\hline \multicolumn{4}{|l|}{ Idade } \\
\hline$\leq 27$ & $1117(31,56)$ & 1305 (33) & $349(42,67)^{b}$ \\
\hline $28-36$ & $1105(31,22)$ & $1415(35)$ & $237(28,97)$ \\
\hline$>36$ & $1317(37,21)$ & $1283(32)$ & $232(28,36)$ \\
\hline \multicolumn{4}{|l|}{ Escolaridade } \\
\hline Analfabeto & $38 \quad(1,07)$ & $61 \quad(2)$ & $17(2,06)^{b}$ \\
\hline Primária & $2283(64,29)$ & $2412(60)$ & $570(69,09)$ \\
\hline Colegial & $1111(31,29)$ & $1238(30)$ & $223(27,03)$ \\
\hline Superior & $119 \quad(3,35)$ & $367 \quad(9)$ & $15 \quad(1,82)$ \\
\hline \multicolumn{4}{|l|}{ Situação conjugal } \\
\hline Casado & $1583(44,58)$ & $1683(41)$ & $317(38,42)^{b}$ \\
\hline Não casado & $1968(55,42)$ & $2395(59)$ & $508(61,58)$ \\
\hline \multicolumn{4}{|c|}{ Nível socioeconômico } \\
\hline Alto & $107 \quad(3,03)$ & $347 \quad(9)$ & $16(1,94)^{b}$ \\
\hline Médio & $1121(31,71)$ & $1256(32)$ & $222(26,97)$ \\
\hline Baixo & $2307(65,26)$ & $2460(61)$ & $585(71,09)$ \\
\hline \multicolumn{4}{|c|}{ Qualificação da ocupação } \\
\hline Alta & $102(2,87)$ & $340 \quad(8)$ & $15(1,82)^{\mathrm{b}}$ \\
\hline Média & $833(23,47)$ & $974(24)$ & $199(24,12)$ \\
\hline Baixa & $2614(73,66)$ & 2762 (68) & $611(74,06)$ \\
\hline
\end{tabular}

a Programa de alimentação oficial do Ministério do Trabalho e Emprego.

b $P<0,05$.

TABELA 2. Prevalências de fatores de risco conforme programa de alimentação ao início do estudo em 8454 trabalhadores da Bahia, Brasil, 1996 a 2000

\begin{tabular}{|c|c|c|c|}
\hline \multirow[b]{3}{*}{ Variáveis } & \multicolumn{3}{|c|}{ Programa de alimentação } \\
\hline & $\begin{array}{c}\text { Programa de } \\
\text { alimentação do } \\
\text { trabalhador }^{2}(n=3551)\end{array}$ & Outro $(n=4078)$ & Nenhum $(n=825)$ \\
\hline & No. (\%) & No. (\%) & No. (\%) \\
\hline Triglicéridos elevados & $356(10,03)$ & $314 \quad(7,70)$ & $48 \quad(5,82)^{b}$ \\
\hline Colesterol total elevado & $1152(32,44)$ & $1234(30,26)$ & $220(26,67)^{c}$ \\
\hline Glicemia elevada & $291 \quad(8,09)$ & $310(7,44)$ & $44(5,07)^{c}$ \\
\hline Hipertensão arterial & $549(15,27)$ & $559(13,42)$ & $88(10,14)^{b}$ \\
\hline Hábito de fumar & $480(13,52)$ & $602(14,76)$ & $133(16,12)$ \\
\hline $\begin{array}{l}\text { Consumo de álcool } \\
\text { Índice de massa corporal }\end{array}$ & $683(19,23)$ & $726(17,80)$ & $331(40,12)^{b}$ \\
\hline Magreza & $111 \quad(3,13)$ & $155 \quad(3,81)$ & $18 \quad(2,18)^{b}$ \\
\hline Eutrofia & $2288(64,60)$ & $2739(67,30)$ & $514(62,38)$ \\
\hline Pré-obesidade & $1143(32,27)$ & $1176(28,89)$ & $292(35,44)$ \\
\hline
\end{tabular}

a Programa de alimentação oficial do Ministério do Trabalho e Emprego.

${ }^{b} P<0,01$.

c $P<0,05$. 
TABELA 3. Associação entre aumento de peso e pré-obesidade e programas de alimentação em 8454 trabalhadores da Bahia, Brasil, 1996 a 2000

\begin{tabular}{lcccc}
\hline \multicolumn{1}{c}{ Variáveis } & $\begin{array}{c}\text { Pessoa- } \\
\text { ano }\end{array}$ & $\begin{array}{c}\text { Taxa de } \\
\text { incidência }\end{array}$ & $\begin{array}{c}\text { Razão de taxa de } \\
\text { incidência bruta } \\
\text { (IC 95\%) }\end{array}$ & RTI $_{\text {aj }}{ }^{a}$ (IC 95\%) \\
\hline $\begin{array}{l}\text { Aumento de peso } \\
\text { PAT }\end{array}$ & 3824 & 0,22 & $2,18(1,76-2,70)$ & $2,21(1,78-2,75)$ \\
$\quad \begin{array}{l}\text { Outro } \\
\text { Nenhum }\end{array}$ & 4410 & 0,25 & $2,54(2,04-3,12)$ & $2,53(2,04-3,13)$ \\
$\begin{array}{l}\text { Pré-obesidade } \\
\quad \text { PAT }\end{array}$ & 906 & 0,10 & 1,00 & 1,00 \\
$\quad$ Outro & 3824 & 0,03 & $1,24(1,01-1,52)$ & $1,35(0,88-2,05)$ \\
$\quad$ Nenhum & 4410 & 0,04 & $1,37(1,11-1,69)$ & $1,36(0,89-2,06)$ \\
1,00 & 1,00 \\
\hline
\end{tabular}

a Razão de taxa de incidência ajustada por idade e sexo.

com PAT foi 0,22 casos/pa; 0,25 casos/ pa para aqueles de empresas com outros programas de alimentação, e 0,10 casos/pa para o grupo sem programa de alimentação (tabela 3). Estimou-se uma associação positiva, estatisticamente significativa, entre aumento de peso e ser trabalhador de empresa com PAT $\left(\mathrm{RTI}_{\text {bruta }}=2,18\right.$; 95\%IC: 1,76-2,70; e RTI ${ }_{a j}=2,21 ; 95 \%$ IC: $\left.1,78-2,75\right)$. Semelhantemente, trabalhadores de empre- sas com outros programas de alimentação tiveram taxa de incidência de aumento de peso duas vezes e meia maior $\left(\mathrm{RTI}_{\text {bruta }}=2,54\right.$; 95\%IC: $2,04-3,12$; $\mathrm{RTI}_{\mathrm{aj}}=2,53$; 95\% $\mathrm{IC}: 2,04-3,13$ ) do que os trabalhadores de empresas sem programas de alimentação. Vale notar que a média de aumento de peso variou de $2,82 \mathrm{~kg}$ / ano (entre trabalhadores cobertos pelo PAT) a 2,78 kg/ano (beneficiários de outros programas de alimentação), com diferenças estatisticamente significativas para a média do grupo de trabalhadores não cobertos por nenhum programa de alimentação, estimada em 2,55 kg/ano (dados não apresentados). Ainda na tabela 3, observou-se também que a taxa de incidência de pré-obesidade foi maior no grupo de trabalhadores com PAT $(0,03$ casos / pa) e outro programa de alimentação $(0,04$ casos / pa) do que entre trabalhadores sem programa de alimentação (0,02 casos/pa). Estas diferenças foram estatisticamente significativas para as medidas brutas (RTI $=1,24$; IC95\%: 1,01-1,52; RTI = 1,37; IC95\%: 1,11-1,69 para trabalhadores de empresas com PAT e outro programa, respectivamente), mas não para as medidas ajustadas por idade e sexo.

Na tabela 4 apresentam-se dados referentes à associação entre programas de alimentação e aumento de peso e pré-obesidade segundo o estado nutricional e o nível socioeconômico dos participantes do estudo. Pode-se observar que os trabalhadores de empresas com PAT ou outros programas de alimentação têm maior taxa de incidência

TABELA 4. Associação entre programa de alimentação e aumento de peso e pré-obesidade conforme o estado nutricional inicial e o nível socioeconômico em 8454 trabalhadores da Bahia, Brasil, 1996 a 2000

\begin{tabular}{|c|c|c|c|c|c|c|}
\hline \multirow[b]{3}{*}{ Variáveis } & \multicolumn{6}{|c|}{ Programa de alimentação } \\
\hline & \multicolumn{2}{|c|}{ Programa de alimentação do trabalhador } & \multicolumn{2}{|c|}{ Outro } & \multicolumn{2}{|c|}{ Nenhum } \\
\hline & Pessoa-ano & $\mathrm{RTI}_{\mathrm{aj}}{ }^{\mathrm{a}}(\mathrm{IC} 95 \%)$ & PA & $\mathrm{RTI}_{\mathrm{aj}}{ }^{\mathrm{a}}(\mathrm{IC} 95 \%)$ & PA & $\mathrm{RTI}_{\mathrm{aj}}^{\mathrm{a}}$ \\
\hline \multicolumn{7}{|c|}{ Aumento de peso } \\
\hline \multicolumn{7}{|c|}{ Estado nutricional inicial } \\
\hline Magreza & 115 & $1,39 \quad(0,72-2,69)$ & 175 & $1,42(0,74-2,70)$ & 24 & 1,00 \\
\hline Eutrofia & 2486 & $1,48(1,26-1,75)$ & 2984 & $1,72(1,46-2,02)$ & 573 & 1,00 \\
\hline Alto & 104 & $1,31 \quad(0,39-4,34)$ & 376 & $1,23(0,39-3,90)$ & 18 & 1,00 \\
\hline Médio & 1262 & $1,77(1,23-2,56)$ & 1389 & $2,19(1,52-3,14)$ & 247 & 1,00 \\
\hline Baixo & 2437 & $2,48(1,89-3,26)$ & 2625 & $2,76(2,10-3,62)$ & 639 & 1,00 \\
\hline \multicolumn{7}{|c|}{ Pré-obesidade } \\
\hline \multicolumn{7}{|c|}{ Estado nutricional inicial } \\
\hline Magreza & 115 & $1,68(0,68-4,15)$ & 175 & $0,70(0,27-1,82)$ & 24 & 1,00 \\
\hline Médio & 1262 & $0,88(0,45-1,71)$ & 1389 & $1,02(0,53-1,95)$ & 247 & 1,00 \\
\hline Baixo & 2437 & $1,65(0,95-2,86)$ & 2625 & $1,55(0,90-2,68)$ & 639 & 1,00 \\
\hline
\end{tabular}

a Razão da taxa de incidência ajustada por idade e sexo. 
de aumento de peso quando se apresentavam com peso normal ou já em estado de pré-obesidade ao início do seguimento. Entre os que eram magros, as associações foram positivas, mas não estatisticamente significativas. Verificou-se também que a medida da associação entre o PAT e aumento de peso se elevava à medida que o nível socioeconômico diminuía, com uma tendência gradual conhecida como gradiente dose-resposta. Tendência semelhante ocorreu para as medidas de associação entre aumento de peso e outros programas de alimentação. Quanto à pré-obesidade, não se encontrou associação estatisticamente significativa com quaisquer tipos de programas de alimentação, ou de acordo com o estado nutricional inicial. Houve uma tendência de associação inversa, não significativa, entre pré-obesidade e programas de alimentação e nível socioeconômico.

\section{DISCUSSÃO}

Os resultados deste estudo fornecem evidências de que, no Brasil, trabalhadores de empresas cobertas pelo PAT ou outros programas similares aumentam de peso em taxas maiores do que os trabalhadores não beneficiários desse programa. Esse aumento de peso ocorreu, especialmente, entre trabalhadores que apresentavam peso normal ou pré-obesidade, ou que pertenciam aos estratos socioeconômicos médio ou baixo ao início do estudo. A taxa de incidência de pré-obesidade, outro indicador do estado nutricional, também foi maior entre os trabalhadores beneficiários de qualquer tipo de programa de alimentação, mas esta diferença desapareceu após o ajuste por sexo e idade. Isso leva a crer que sexo e idade são preditores mais importantes de pré-obesidade do que ser beneficiário de programas de alimentação. Semelhantemente ao ganho de peso, houve maior incidência de pré-obesidade entre os trabalhadores de nível socioeconômico médio e baixo, embora essas diferenças não tenham sido estatisticamente significativas. Estes achados demonstram que, nas empre- sas onde foram implantados programas de alimentação, houve um efeito não desejado sobre o estado nutricional dos trabalhadores, ou seja, um aumento de peso naqueles que eram eutróficos ou que já eram pré-obesos, e não, como seria desejável, apenas entre os trabalhadores com peso abaixo do normal.

Embora o PAT tenha sido, em sua origem, destinado a atingir prioritariamente as classes pobres, onde prevaleceria o peso abaixo do ideal e a má nutrição, o ganho de peso não foi maior entre os trabalhadores magros, mas sim entre os que já tinham préobesidade. Além disso, o aumento de peso entre os trabalhadores eutróficos ou pré-obesos ocorreu especialmente entre os grupos mais pobres. Vale notar que os dados deste estudo se referem a taxas estimadas para a unidade de variação do tempo de seguimento (ano), e que, como o aumento de peso é freqüentemente cumulativo, a pré-obesidade ou a obesidade poderão se instalar se o trabalhador permanecer no programa de alimentação por alguns anos.

A interpretação adequada destes achados requer conhecimentos de outros campos disciplinares ainda escassos para o contexto e população focalizados neste estudo. Uma das possíveis explicações para os efeitos observados dos programas de alimentação é o perfil de classe dos hábitos alimentares. Em um estudo conduzido na França (12), verificou-se que a classe proletária consumia mais alimentos tidos como "fortes", enquanto a pequena burguesia escolhia mais alimentos "saudáveis". Nem a renda nem o preço do alimento se associavam ao tipo de alimento consumido. No Brasil, encontrou-se que a população de baixo poder aquisitivo dava preferência ao consumo de cereais e leguminosas, e a de renda mais alta, aos vegetais e carnes (13). Esses dados são sugestivos de uma noção aparentemente comum nos segmentos pobres da população, de que o bom alimento é aquele que dá "sustentação ao corpo para o trabalho". Desse modo, trabalhadores pobres, entre os quais predominam as atividades braçais, de grande gasto energético, prefeririam alimentos provedores de "sustança", considerados fortes, a exemplo do feijão com arroz ou farinha. ${ }^{4}$ Além disso, a sustança é também referida, no contexto doméstico familiar das camadas populares de várias regiões do país, como uma propriedade da alimentação mais adequada para o homem, cuja atividade física é mais comumente pesada, do que para as mulheres e crianças (14).

Assim, a sensação de saciedade dada por alimentos ricos em fibras e de custo mais barato, e a compreensão de que seriam fontes de maior energia, poderiam justificar a escolha de alimentos que proporcionam um peso corporal distante da eutrofia. Como a refeição provida pela empresa é entendida como um benefício, pode-se pensar que o trabalhador tende a ingerir mais alimentos de modo a obter uma compensação ainda maior por parte daqueles que se apropriam da sua força de trabalho, em especial através de baixos salários. ${ }^{4}$ Embora esses estudos tenham sido realizados nos anos 1970 e 1980, não há evidências de que a realidade examinada nesta investigação seja diferente. Mais recentemente, verificou-se que pessoas pobres, para as quais não há garantia de acesso cotidiano à comida, procuram, sempre que lhes é dada a oportunidade de acesso, consumir uma quantidade maior do que a necessária (15).

Vale também ressaltar que o maior aumento de peso entre indivíduos préobesos encontrado neste estudo pode não estar relacionado diretamente à ingestão calórica, mas à baixa atividade física espontânea, que pode permitir um balanço energético positivo e, conseqüentemente, aumento de peso nas pessoas propensas à obesidade. Estudos desenvolvidos na década de 1970 mostraram haver gasto energético decorrente da atividade física espontânea que variava entre 100 e $800 \mathrm{kcal} /$ dia. Indivíduos obesos eram menos ativos do que os seus companheiros

\footnotetext{
4 Woortmann K. Hábitos e tabus alimentares em populações de baixa renda [documento mimeografado]. Distrito Federal: Universidade de Brasília; 1978.
} 
magros, que tinham, portanto, um gasto calórico maior (16). Assim, os pré-obesos teriam uma maior tendência ao aumento de peso do que os eutróficos ou magros.

Os achados desta pesquisa são consistentes com os encontrados nos poucos estudos de avaliação do impacto do PAT, embora as comparações estejam limitadas pelos seus propósitos e desenhos distintos. Destacam-se, neste sentido, estudos sobre a oferta calórica dos cardápios do programa, como o de Moura (17), no qual se estimou que $37 \%$ dos cardápios de empresas registradas no PAT apresentavam valor calórico igual ou superior a $1400 \mathrm{kcal}$. Isto é reiterado pelo estudo de Gambardella (18) que, observando cardápios propostos pelo PAT, estimou uma variação de 1400 a $2200 \mathrm{kcal}$, sendo que $66 \%$ apresentavam valores superiores a $1600 \mathrm{kcal}$. Com base no cálculo das necessidades diárias dos trabalhadores, a autora estimou que apenas uma refeição cobriria cerca de 40 a $60 \%$ destas necessidades para o sexo masculino, e 60 a $80 \%$ para as mulheres trabalhadoras. Tais dados são sugestivos de que o PAT, embora alcance sua meta nutricional de oferta de refeições "maiores", com um mínimo de 1400 kcal, representa uma excessiva oferta calórica que se concentra em uma única refeição. Possivelmente, esta é uma das razões para os efeitos não desejados observados neste estudo, como ganho de peso e aumento da pré-obesidade entre adultos eutróficos e pré-obesos ou de baixo nível socioeconômico. Como as estratégias empregadas nos demais programas de alimentação são desconhecidas e não uniformes, não é possível generalizar essas explicações.

Ademais, como houve ganho de peso, que cumulativamente pode levar à obesidade, conhecido fator de risco para doenças crônico-degenerativas, especialmente as cardiovasculares, o PAT ou outros programas de alimentação podem, ao contrário do previsto, ter contribuído para o aumento da morbidade entre os trabalhadores beneficiados. Dessa forma, seria plausível um aumento dos problemas de saúde e, conseqüentemente, de licenças médi- cas. Em apoio a essa hipótese, Campino et al..$^{5}$ encontraram um número maior de dispensas médicas concedidas a trabalhadores após a implantação do PAT, comparando-se com igual período anterior; e Moura (17) verificou que o número de dias de licença médica de trabalhadores beneficiados pelo PAT era maior do que entre aqueles não inscritos no programa.

A compreensão dos resultados deste estudo também se assenta na confrontação com cada um dos componentes do PAT, enquanto política social e programa setorial. O objetivo do PAT é suprir as necessidades alimentares do corpo do trabalhador, enquanto força de trabalho que produz valor. Tratase, portanto, de uma concepção funcionalista e instrumental do corpo, que não reconhece o sentido simbólico e cultural de que se reveste a alimentação. Uma alimentação adequada envolve não apenas o acesso, mas a relação entre indivíduo e alimento, inclusive suas representações sociais, comportamentos, conhecimento e capacidade de escolha, entre outros aspectos. Assim, a racionalidade biológica implícita no PAT e outros programas de alimentação se enfrenta com o trabalhador visto e pensado em sua totalidade, uma vez que o comportamento alimentar orienta-se por outras racionalidades $(12,19)$.

O PAT almejava, desde as origens, superar a desnutrição calórica dos trabalhadores, fundamentando-se em dados de um estudo conduzido pela Fundação Getúlio Vargas, que mostrava nas populações de baixa renda uma ingestão calórica de apenas 60\% das necessidades nutricionais (20). Embora não seja possível contestar a veracidade desse dado, o que se pode argumentar é que o PAT não beneficia apenas trabalhadores de baixa renda, ou braçais. Além disso, há indícios de que o perfil nutricional dos trabalhadores tenha se modificado neste pe-

\footnotetext{
5 Campino ACC, Cacciamali C, Cyrillo D. Avaliação sócio-econômica do programa de alimentação do trabalhador [documento mimeografado]. Conselho Nacional de Desenvolvimento Científico e Tecnológico e Fundação Instituto de Pesquisas Econômicas 1982;1:1-35.
}

ríodo de existência do PAT. Por exemplo, um estudo mostra que houve um aumento substancial do poder de compra da cesta básica por assalariados após a implantação de um programa de estabilização econômica iniciado em 1994, denominado Plano Real (21). Os resultados da Pesquisa Nacional sobre Saúde e Nutrição (PNSN) (22) e Pesquisa de Padrão de Vida (PPV) (23), quando comparados ao Estudo Nacional da Despesa Familiar (ENDEF), demonstram que houve uma redução do baixo peso e aumento da pré-obesidade na população adulta no país. Ainda que esse efeito possa ser também conseqüência da implantação do PAT, mudanças semelhantes no perfil nutricional da população têm ocorrido em todo o mundo. Apesar de o Ministério do Trabalho e Emprego não ter avaliado o impacto do PAT nem monitorado o perfil nutricional dos trabalhadores, recentemente foi introduzido um ajuste admitindo valores mínimos (1 200 kcal) e máximos (1 600 kcal) para as refeições maiores, a depender do nível de atividade física da ocupação do trabalhador (24).

Um outro problema que o PAT pretendia superar era a baixa produtividade dos trabalhadores, pensada como decorrente do déficit alimentar. Recuperando-se o bom estado nutricional dos trabalhadores haveria resultante aumento da produtividade. Essa afirmação se respalda em estudos que demonstram a relação entre alimentação e produtividade $(25,26)$. Campino et al., 5 por exemplo, verificaram que, em empresas com o PAT, houve redução em indicadores indiretamente relacionados à produtividade, como os índices de acidente de trabalho, taxas de absenteísmo e rotatividade. Em um outro estudo (17), verificou-se que o PAT reduzia apenas as taxas de absenteísmo. Os resultados deste estudo limitam-se ao estado nutricional e, portanto, não permitem conclusões sobre o impacto do PAT na produtividade, embora seja implausível pensar que aumento de peso e pré-obesidade estejam favorecendo a produtividade.

Infelizmente, o PAT não contemplou, originalmente, a educação nutricional participativa nas suas diretrizes. 
Essa lacuna só recentemente começou a ser preenchida. Uma cartilha sobre educação alimentar elaborada pelo Ministério do Trabalho e Emprego foi distribuída, mas o seu impacto sobre mudanças comportamentais de massa é reconhecidamente questionável. Um outro aspecto também relacionado à estratégia do PAT é a generalização das recomendações, direcionadas a todos os trabalhadores, independentemente do estado nutricional, não focalizando grupos de risco. Como se trata de uma recomendação geral para uma população reconhecidamente heterogênea, não chegam a surpreender os seus resultados contraditórios.

A principal limitação do presente estudo provém da utilização de dados secundários, seguida pelas medidas auto-referidas de peso e altura. Tam- pouco estavam disponíveis alguns dados relevantes para a análise, como a composição da dieta e grau de consumo energético da atividade ocupacional. Todavia, por serem dados informatizados, os registros foram padronizados e completos, e a sistematização ao longo do tempo permitiu que pudesse ser construída a coorte retrospectiva. Medidas auto-referidas de peso e altura são cada vez mais empregadas em pesquisas, uma vez que os estudos de validade mostram alta correlação destas com medidas diretamente aferidas $(27,28)$. Este estudo apresenta vantagens em relação a outras pesquisas avaliativas do PAT em função de seu desenho longitudinal com uma população que permite atingir o poder estatístico necessário para a análise da hipótese em questão.

\section{REFERÊNCIAS}

1. Brasil. Decreto-lei No. 77.116, Diário Oficial da União 1976 fevereiro 6.

2. Brasil. Lei No. 6.321, de 14 de abril de 1976. Diário Oficial da União 1976 abril 19.

3. Brasil. Portaria No. 652, Diário Oficial da União 1976 dezembro 22.

4. Molina M, Del CB. Perfil nutricional de trabalhadores da Companhia Siderúrgica de Tubarão, ES. Em: Anais do XV Congresso Brasileiro de Nutrição. Brasília: Associação Brasileira de Nutrição; 1997. p. 58.

5. Ell E, Camacho LAB, Chor D. Perfil antropométrico de funcionários de banco estatal no Estado do Rio de Janeiro/Brasil: I —índice de massa corporal e fatores sóciodemográficos. Cad Saude Publica 1999;15(1): 113-121.

6. World Health Organization. Obesity: preventing and managing the global epidemic. Geneva: WHO; 1997.

7. The sixth report of the Joint National Committee on prevention, detection, evaluation, and treatment of high blood pressure. Arch Int Med 1997;157(21):2413-2446.

8. Sociedade Brasileira de Cardiologia. Segundo Consenso Brasileiro sobre Dislipidemia: detecção, avaliação e tratamento. Arq Bras Cardiol 1996;67(2):1-16.

9. Miller O. Laboratório para o clínico. Rio de Janeiro: Livraria Atheneu Editora; 1991.

10. SAS. SAS/STAT - User's guide. Version 6. 4th ed. Vol. 2. Cary, NC: SAS Institute Inc.; 1989.

11. Rothman KJ, Greenland S. Modern epidemiology. 2nd ed. Philadelphia: Lippincott-Raven Publishers; 1998.

12. Boltanski L. As classes sociais e o corpo. 2a ed. Rio de Janeiro: Graal; 1984.

13. Alves ELG. Desnutrição e pobreza no Brasil: algumas evidências. Cad Pesquisa (Fundação Carlos Chagas) 1979;29(6):77-86.
14. Zaluar A. As mulheres e a direção do consumo doméstico (estudo de papéis familiares nas classes populares urbanas). Em: Colcha de Retalhos: estudos sobre a família no Brasil. São Paulo: Editora Brasiliense; 1982.

15. Freitas MCS. Significados da fome: um estudo etnográfico em um bairro popular de Salvador [tese de doutorado]. Bahia: Instituto de Saúde Coletiva, Universidade Federal da Bahia;2000.

16. Napoli R, Horton ES. Necesidades energéticas. Em: Ekhard E, Ziegler Y, Ljfiler JR. Conocimientos actuales sobre nutrición. Washington DC: Organización Panamericana de la Salud e Instituto Internacional de Ciencias de la Vida; 1997. (Publicación Científica 565:1-7).

17. Moura JB. Avaliação do programa de alimentação do trabalhador no Estado de Pernambuco, Brasil. Rev Saude Publica 1986;20(2): 115-128.

18. Gambardella AMD. O programa de alimentação do trabalhador frente às recomendações nutricionais para esse segmento específico da população: área metropolitana de São Paulo [dissertação de mestrado]. São Paulo: Faculdade de Saúde Pública, Universidade de São Paulo; 1990

19. Food and Agriculture Organization of the United Nations. Preparation and use of food based dietary guidelines [site na Internet]. Disponível em: http://www.fao.org/docrep/ x0243e/x0243e09.htm\#P1489_136013. Acessado 14 de dezembro de 2001.

20. Ministério do Trabalho. Incentivos fiscais para a alimentação do trabalhador. Coleção VII Promoção Social. Brasília: Ministério do Trabalho; 1979.

21. Brasil. Presidente (1999-2002: FH Cardoso) 7 anos do real: estabilidade, crescimento e desenvolvimento social [coleção documentos da
Apesar dos limites das ações do PAT, há evidências de grande aceitação por parte dos trabalhadores, que vêem o programa como uma compensação salarial (29). Dada a penúria em que vive grande parte dos trabalhadores do país, a relevância social de um programa como o PAT é inquestionável. Todavia, os resultados desta pesquisa indicam que as ações do PAT necessitam de revisão. Uma vez reformulado, recomenda-se a sua ampliação para o total dos trabalhadores do país, especialmente aqueles do setor informal, que representam mais da metade do mercado de trabalho e incluem as camadas mais pobres da população. Finalmente, como um dos componentes das políticas sociais, o PAT deve também estar articulado aos programas de prevenção de riscos e de promoção da saúde na empresa. presidência da república]. Disponível em: http://www.presidencia.gov.br/publi_04/7 anos.pdf. Acessado 14 de dezembro de 2001.

22. Coitinho DC, Leão MM, Recine E, Sichieri R. Pesquisa Nacional sobre Saúde e Nutrição: condições nutricionais da população brasileira: adultos e idosos. Brasília: PNSN/ IBGE; 1991.

23. Pesquisa de padrão de vida. PPV aprofunda investigação de indicadores sociais [site na Internet]. Disponível em: http://www. ibge. gov.br/imprensa/Noticias/ppv 11.htm. Acessado 17 de outubro de 1998.

24. Brasil. Portaria Interministerial no. 05 , de 30 de novembro de 1999.

25. Maturu NR. Alimentación y productividad. Rev Int Trabajo 1979;98(1):51-62.

26. Gomes JR. Alimentação e trabalho. Rev Bras Saude Ocupacional 1982;40(10):12-15.

27. Izquierdo J, Vioque J. Validez de los datos antropométricos declarados para la determinación de la prevalencia de obesidad. Med Clin 1996;106(19):725-729.

28. Chor D, Coutinho ESF, Laurenti R. Reliability of self-reported weight and height among State bank employees. Rev Saude Publica 1999;33(1):16-23.

29. Viana $S V$. Indústria moderna e padrão alimentar: o espaço do trabalho, do consumo e da saúde [tese de doutorado]. Bahia: Instituto de Saúde Coletiva, Universidade Federal da Bahia; 2000.

Manuscrito recibido el 18 de julio de 2001 y aceptado para publicación, tras revisión, el 18 de diciembre de 2001. 
ABSTRACT Objective. The worker food program of the Brazilian Ministry of Labor and Employment attempts to ensure adequate nourishment for low-income workers. This study evaluates the nutritional impact of the program, which currently serves approximately 10 million workers.

\section{Impact of the worker food program in Brazil}

Methods. This retrospective dynamic cohort study was carried out with 8454 workers living in the state of Bahia, Brazil. Health-related data from January 1996 through April 2000 were obtained from computerized medical records from an organization that provides health surveillance services to a large number of companies in the state. The analysis utilized demographic data and nutritional data (weight and also preobesity, that is, a body mass index of 25.00-29.99). Additional information was obtained from the companies through telephone interviews.

Results. Being covered by the food program was positively associated with weight gain (age- and sex-adjusted incidence-density ratio $=2.21 ; 95 \%$ confidence interval: 1.78-2.75). No statistically significant association was found between the program and preobesity. The risk of weight gain was higher $(P<0.05)$ among workers who, at baseline, were of normal weight (body mass index of 18.50-24.99), were preobese, or were from a low socioeconomic background.

Conclusions. These results suggest that the worker food program has an unintended negative impact on the nutritional status of workers from a low socioeconomic background. The program's approach, which is limited to dietary recommendations concerning the caloric content of meals, should be revised to better promote the health of the workers participating in the program.

\section{Clasificación Internacional de Enfermedades Aplicada a Odontología y Estomatología (CIE-OE), 3. ${ }^{a}$ ed.}

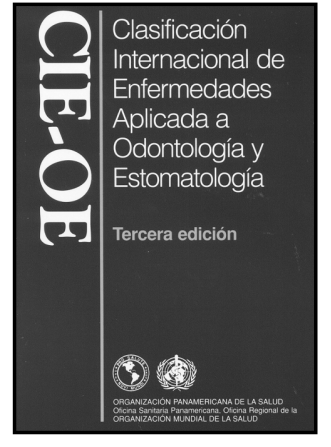

1996, $238 \mathrm{pp}$., ISBN 9275315620 Código: PC 562,

Precio: US\$ 32.00/ US\$ 22.00 en América Latina y el Caribe
Esta tercera edición de la Clasificación Internacional de Enfermedades Aplicada a Odontología y Estomatología (CIE-OE) tiene por objeto proveer bases prácticas y convenientes para codificar y clasificar datos relativos a los trastornos bucales y dentarios. La CIE-OE se deriva directamente de la Décima Revisión de la Clasificación Internacional de Enfermedades (CIE-10), y ha sido concebida como parte de la "familia"de clasificaciones de enfermedades y problemas relacionados con la salud.

En la CIE-OE se incluyen todas las enfermedades y trastornos que se presentan en la cavidad bucal y estructuras adyacentes, tienen manifestaciones en ellas o están asociadas con las mismas. La mayoría de las clasificaciones provistas por la CIE-10 han sido subdivididas y expandidas para incluir un quinto carácter. El propósito de la inclusión de este quinto carácter es llamar la atención de los profesionales de la salud bucal sobre la necesidad de realizar un diagnóstico detallado para cada paciente a fin de proveer un registro estandarizado para todos los diagnósticos y facilitar la comparación de los datos a nivel internacional. Se espera que la CIE-OE contribuya en forma importante a la recolección de datos epidemiológicos sobre las enfemedades bucales menos frecuentes, para las cuales los métodos de vigilancia son impracticables. 